\title{
ON PLANE RIGID FRAMES LOADED PERPENDICULARLY TO THEIR PLANE*
}

BY

W. PRAGER (Brown University) AND G. E. HAY (University of Michigan)

1. Introduction. For purposes of stress analysis, the engineer prefers to consider his structures as consisting of plane systems, each of which is subject to forces acting in its plane. A typical example is furnished by the conventional analysis of a parallel chord bridge span in which the side trusses take the vertical loads and the top and bottom trusses the transverse loads due to wind, etc. In civil engineering this resolution of space systems into plane components is possible in most cases, and only very rarely is a structure considered as a unit in space. Accordingly, the methods of dealing with space structures have not been developed nearly as much as those used in the analysis of plane structures. Of course, the general principles of structural theory, for instance the principle of virtual work or Castigliano's principle, apply to space structures as well as to plane structures but, as is known from

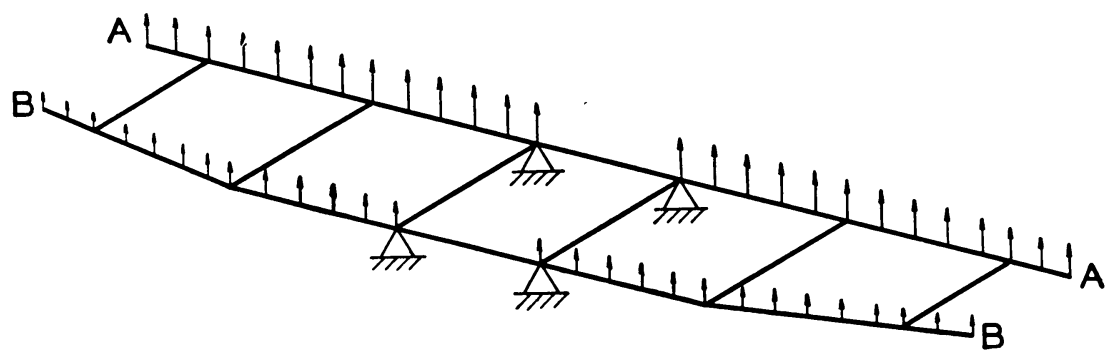

FIG. 1a. Two-sparred wing.

the case of plane structures, these principles frequently do not offer the most convenient approach to the solution of a particular problem. As regards special methods, which have been developed so abundantly in the case of plane structures, little work has as yet been done in the field of space structures. Most of this work is concerned with pin-jointed frameworks. The iteration procedure of $\mathrm{R}$. V. Southwell's relaxation method can be applied to space structures as well as to plane structures, ${ }^{1}$ but efficient direct methods for the stress analysis of rigid frames in space are entirely lacking. The present paper, intended as a contribution towards the development of such methods, deals with the particular case of plane rigid frames carrying loads which act per-

* Received Dec. 3, 1942.

${ }^{1}$ See R. V. Southwell: Relaxation methods in engineering science, Chap. IV, Oxford 1940. 
pendicularly to the plane of the frame. An example of this type of structure is the monoplane wing of Fig. 1a, where the spars $\mathrm{A}-\mathrm{A}$ and $\mathrm{B}-\mathrm{B}$ are connected by several main ribs which are fastened rigidly to the spars. Another example is the foot ring of an observatory cupola shown in Fig. 1b.

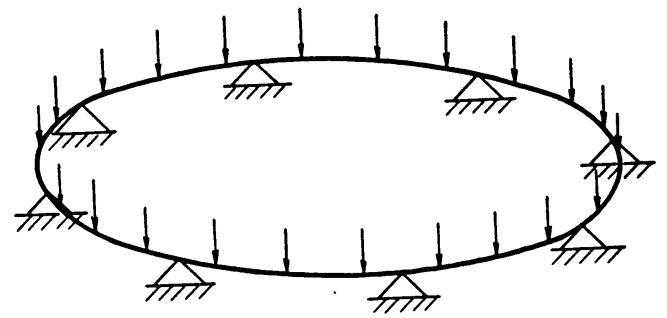

Fig. 1b. Cupola foot ring.

The method proposed in this paper makes extensive use of a dual analogy between plane structures loaded in their plane and plane structures loaded perpendicularly to their plane. In the case of a single straight beam this analogy forms the basis of the method of conjugate beams ${ }^{2}$ which can be considered as a par-

ticular case of the present method.

2. Definitions, notations and sign conventions. This paper is concerned with rigid frames consisting of straight or curved members whose axes lie in the same plane. This plane is called the structural plane. We will consider only frames with members such that every cross section has a principal axis of inertia at its centroid lying in the structural plane. Accordingly, when the frame is subject to forces acting in the structural plane, the points on the axis of any member remain in the structural plane. On the other hand, when the frame is loaded perpendicularly to its plane, the displacements of the points on the axis of any member are normal to the structural plane. For conciseness, the first type of loading will be referred to as plane loading and the second as space loading.

In the case of plane loading the stresses transmitted across any cross section of a member of the frame are statically equivalent to the following stress resultants: 1) an axial force which, for the sake of brevity, will be called the pull although it may produce either compression or tension; 2) a transverse force, called the shear, which acts in the structural plane normal to the axis of the member under consideration; 3) a couple, called the bending moment, which also acts in the structural plane. In the case of space loading the stress resultants are: 1) a twisting couple, called torque, acting in the plane of the cross section; 2) a bending couple, called bending moment, whose plane is perpendicular to the structural plane as well as to the cross-sectional plane; 3 ) a transverse force, called shear, whose line of action is normal to the structural plane.

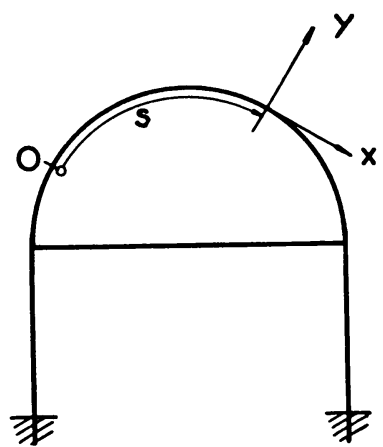

FIG. 2.

${ }^{2}$ H. M. Westergaard, Deflection of beams by the conjugate beam method, Journal of the Western Society of Engineers, 26, 369 (1921). 
In order to arrive at notations applicable to both types of loading we choose on the axis of each member of the frame an origin $\mathrm{O}$ and denote by $s$ the arc length of the axis measured from this point. A cross section $B$ is then specified by giving the corresponding value of $s$ (Fig. $2^{*}$ ). In most cases it will be convenient to choose the origin $\mathrm{O}$ at one end of the member, in which case $s$ will have positive values only. In order to establish appropriate sign conventions for the loads, displacements, stress resultants and distortions at the cross section $B$, we introduce a rectangular right hand triad with origin at $B$, the $x$-axis being tangent to the axis of the member at $\mathrm{B}$ in the direction of increasing $s$, and the $y$-axis lying in the structural plane (Fig. 2).

The loads which the structure carries at B may be forces or couples, either concentrated or distributed, or both. The components of the concentrated force at B, the distributed force at B, the concentrated couple at B and the distributed couple at B we denote by $F_{x}, F_{y}, F_{z}, f_{x}, f_{y}, f_{z}, C_{x}, C_{y}, C_{z}, c_{x}, c_{y}, c_{z}$ respectively, relative to the rectangular triad at $\mathrm{B}$. For example, $C_{x}$ is a concentrated twisting couple, and is positive if its sense is the same as that of the $90^{\circ}$ rotation necessary to move the $y$-axis in to coincidence with the $z$-axis. Not all the load components thus defined have practical importance; however, the analogies which we intend to establish appear more clearly when the most general case is considered.

The force system transmitted across the cross section at $B$ is equivalent to a force at the centroid plus a couple. These will be referred to as the stress resultants, and we shall denote their components by $R_{x}, R_{y}, R_{z}, M_{x}, M_{y}, M_{z}$ respectively, relative to the rectangular triad at B. $R_{x}$ is the pull, $R_{y}$ and $R_{z}$ the shears parallel and perpendicular to the structural plane, $M_{x}$ the torque and $M_{y}, M_{z}$ the bending moments.

The stress resultants and the loads are connected by the equations of equilibrium. If no concentrated forces are applied at the cross section $\mathrm{B}$, the equations of equilibrium for a straight structural member are:

$$
\begin{array}{ll}
R_{x}^{\prime}+f_{x}=0, & M_{x}^{\prime}+c_{x}=0, \\
R_{y}^{\prime}+f_{y}=0, & M_{y}^{\prime}+c_{y}-R_{z}=0, \\
R_{z}^{\prime}+f_{z}=0, & M_{z}^{\prime}+c_{z}+R_{y}=0,
\end{array}
$$

where the dashes denote differentiation with respect to the arc length $s$. If concentrated loads $F_{x}, F_{y}, F_{z}$ and $C_{x}, C_{y}, C_{z}$ are applied at $\mathrm{B}$, we have

$R_{x}(s+\epsilon)-R_{x}(s-\epsilon)+F_{x}=0, \quad M_{x}(s+\epsilon)-M_{x}(s-\epsilon)+C_{x}=0$,

$R_{y}(s+\epsilon)-R_{y}(s-\epsilon)+F_{y}=0, M_{y}(s+\epsilon)-M_{y}(s-\epsilon)+C_{y}=0$,

$R_{z}(s+\epsilon)-R_{z}(s-\epsilon)+F_{z}=0, M_{z}(s+\epsilon)-M_{z}(s-\epsilon)+C_{z}=0$,

where $\epsilon$ denotes an arbitrarily small length.

* In Fig. 2 the origin of the system $x, y$ should be marked B. 
The displacement of the cross section $\mathrm{B}$ is specified by the components $u_{x}, u_{y}, u_{z}$ of the translation of the centroid and the components $\theta_{x}, \theta_{y}, \theta_{z}$ of the rotation of the cross section.

Finally, the six distortion components, $g_{x}, g_{y}, g_{z}$ and $h_{x}, h_{y}, h_{z}$, of a straight structural member are defined as follows:

$$
\begin{array}{ll}
g_{x}=u_{x}^{\prime}, & h_{x}=\theta_{x}^{\prime}, \\
g_{y}=u_{y}^{\prime}-\theta_{z}, & h_{y}=\theta_{y}^{\prime}, \\
g_{z}=u_{z}^{\prime}+\theta_{y}, & h_{z}=\theta_{z}^{\prime},
\end{array}
$$

where the dashes again denote differentiation with respect to the arc length $s$. $g_{x}$ will be called the stretch, $g_{y}$ and $g_{z}$ the slips, $h_{x}$ the twist and $h_{y}, h_{z}$ the bends. Two structural members may be connected by a link which permits some relative displacement of the end sections of the two members, for instance by a hinge permitting a free bend. Such relative displacements can be handled as concentrated distortions:

$$
\begin{array}{ll}
G_{x}=u_{x}(s+\epsilon)-u_{x}(s-\epsilon), & H_{x}=\theta_{x}(s+\epsilon)-\theta_{x}(s-\epsilon), \\
G_{y}=u_{y}(s+\epsilon)-u_{y}(s-\epsilon), & H_{y}=\theta_{y}(s+\epsilon)-\theta_{y}(s-\epsilon), \\
G_{z}=u_{z}(s+\epsilon)-u_{z}(s-\epsilon), & H_{z}=\theta_{z}(s+\epsilon)-\theta_{z}(s-\epsilon),
\end{array}
$$

where $\epsilon$ is again an arbitrarily small length.

For elastic structural members the stress resultants can be represented as the products of the corresponding distortions and stiffness factors:

$$
\begin{aligned}
& R_{x}=\alpha_{x} g_{x}, \quad R_{y}=\alpha_{y} g_{y}, \quad R_{z}=\alpha_{z} g_{z}, \\
& M_{x}=\beta_{x} h_{x}, \quad M_{y}=\beta_{y} h_{y}, \quad M_{z}=\beta_{z} h_{z},
\end{aligned}
$$

where $\alpha_{x}=E A, \alpha_{y}=G A / k_{y}, \alpha_{z}=G A / k_{z}, \beta_{x}$ is the torsional rigidity of the member, $\beta_{y}=E I_{z}, \beta_{z}=E I_{y}, E$ being Young's modulus, $A$ the area of the cross section, $G$ the modulus of rigidity, $k_{y}$ and $k_{z}$ constants depending on the shape of the cross section, $I_{y}$ and $I_{z}$ the moments of inertia of the cross section with respect to the axes of $y$ and $z$.

3. Analogy between statics of plane loaded and kinematics of space

\begin{tabular}{|c|c|}
\hline Statics of Plane Loaded Frame & Kinematics of Space Loaded Frame \\
\hline $\begin{array}{l}\text { Loads }\left\{\begin{array}{lll}F_{x}, & F_{y}, & C_{z} \\
f_{x}, & f_{y}, & c_{z}\end{array}\right. \\
\text { Stress resultants } R_{x}, R_{y}, M_{z}\end{array}$ & $\begin{array}{l}\text { Distortions }\left\{\begin{array}{rrr}-H_{x}, & -H_{y}, & -G_{z} \\
-h_{x}, & -h_{y}, & -g_{z}\end{array}\right. \\
\text { Displacements } \begin{array}{rrrr}\theta_{x}, & \theta_{y}, & u_{z}\end{array}\end{array}$ \\
\hline
\end{tabular}
loaded frames. For a plane loaded frame the loads $F_{z}, f_{z}, C_{x}, c_{x}, C_{y}, c_{y}$ and the stress resultants $R_{z}, M_{x}, M_{y}$ vanish. Similarly, for a space loaded frame the displacements $u_{x}, u_{y}, \theta_{z}$ and the distortions $G_{x}, g_{x}, G_{y}, g_{y}, H_{z}, h_{z}$ are zero. The remaining equations (1) for the members of the plane loaded frame then are seen to correspond exactly to the remaining equations (2) for the members of the space loaded frame according to the Table I.

TABLE I 
At a point $B$ where two straight members are rigidly fastened to one another under the angle $\phi$ (Fig. 3 ), we have the following relations between the stress resultants of the plane loaded frame at the two sides of $\mathrm{B}$ :

$$
\bar{R}_{x}=R_{x} \cos \phi+R_{y} \sin \phi, \quad \bar{R}_{y}=-R_{x} \sin \phi+R_{y} \cos \phi, \quad \bar{M}_{z}=M_{z} .
$$

These relations correspond exactly to the following relations for the displacement components of the space loaded frame on the two sides of $\mathrm{B}$ :

$\bar{\theta}_{x}=\bar{\theta}_{x} \cos \phi+\theta_{y} \sin \phi, \quad \bar{\theta}_{y}=-\theta_{x} \sin \phi+\theta_{y} \cos \phi, \quad \bar{u}_{z}=u_{z}$.

A curved member may be considered as the limiting case of a polygonal arrangement of straight members. Accordingly, the correspondence between the stress resultants of the plane loaded frame and the displacements of the space loaded frame remains valid in the case of frames containing curved members. Of course, equations (1) and (2) in their present forms do not apply to frames with curved members. Equations applicable to such frames will be developed in a later paper.

If more than two members join at the same point, the analogy breaks down. This can be seen from the example in Fig. 4, where we have for the stress resultants of the plane loaded frame

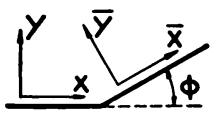

FIG. 3.

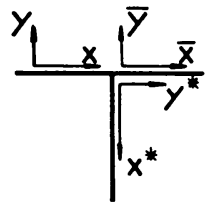

Fig. 4.

$$
\bar{R}_{x}=R_{x}-R_{y}^{*}, \quad \bar{R}_{y}=R_{y}+R_{x}^{*}, \quad \bar{M}_{z}=M_{z}-M_{z}^{*},
$$

and for the displacements of the space loaded from

$$
\bar{\theta}_{x}=\theta_{x}=\theta_{y}^{*}, \quad \bar{\theta}_{y}=\theta_{y}=-\theta_{x}^{*}, \quad \bar{u}_{z}=u_{z}=u_{z}^{*} .
$$

The analogy therefore applies directly only to frames which consist of a simple chain of members without branch points. In spite of this fact the

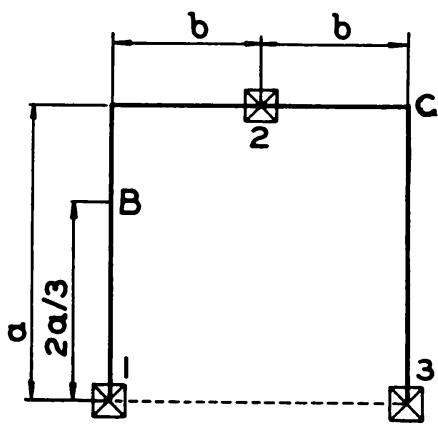

FIG. 5. analogy is very useful even in the case of more complex frames since these may be considered as consisting of simple frames to which the analogy can be applied.

In order that the analogy indicated above hold everywhere in the structures, it is necessary that there can be a certain correspondence between the various supports and links in the frame with plane loading and the frame with space loading. For example, the analogy is maintained if to a pin support in the frame with plane loading there corresponds a simple support in the plane with space loading. A consideration of certain types of supports and links leads to the results presented in Table II. This table is by no means complete. 


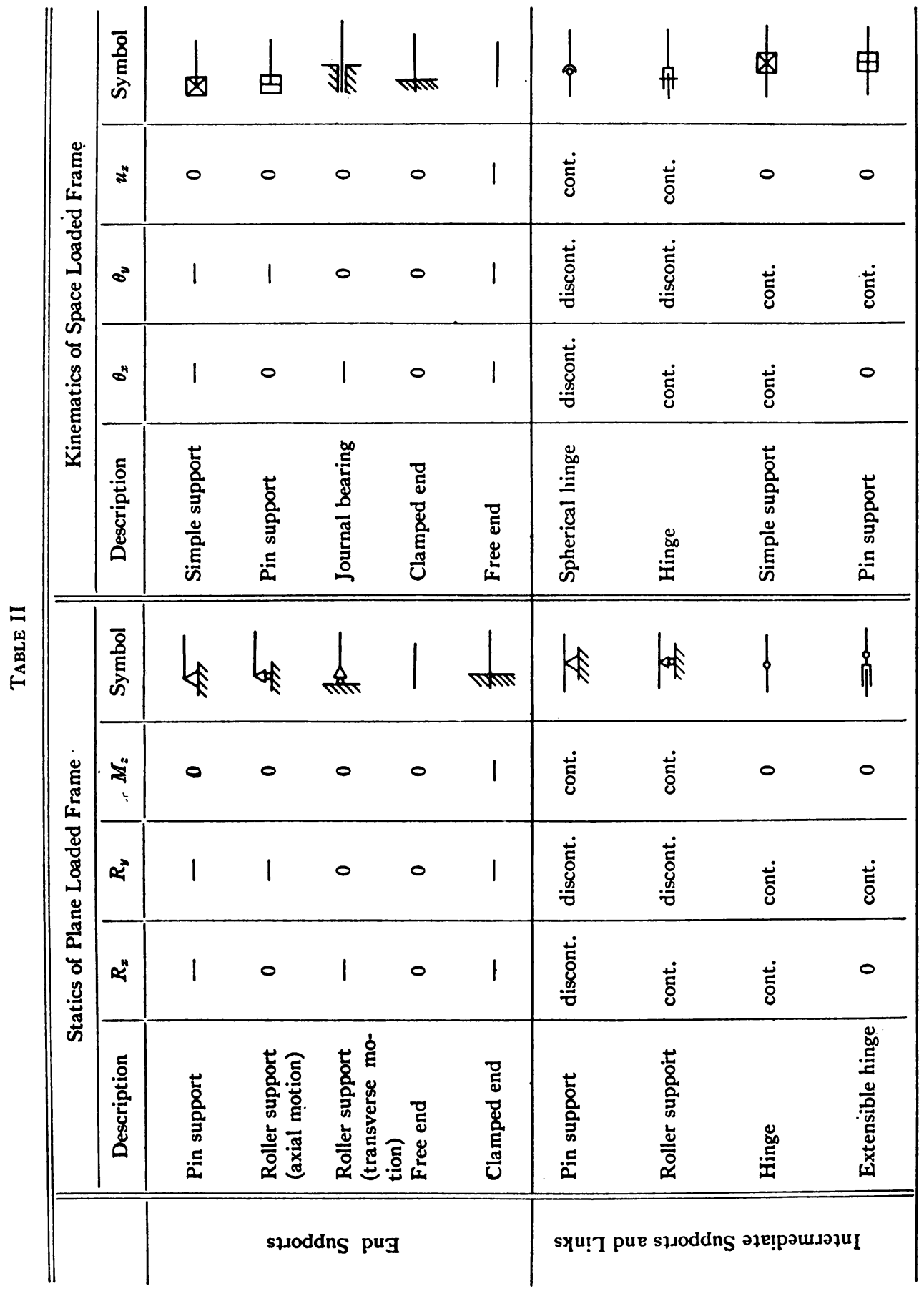


We shall now use this analogy to determine the influence lines for the stress resultants of a statically determinate space loaded frame. We consider the rectangular frame with three pin supports 1, 2, 3 shown in Fig. 5. According to a well known principle of the theory of structures, the ordinates of the influence line for the bending moment produced at the section $\mathrm{B}$ by transverse loads $F_{z}$ are precisely the displacements $u_{z}$ produced by a unit bend, $H_{y}=1$, at $\mathrm{B}$. According to the analogy explained above these displacements can be obtained as the bending moments, $M_{z}$, produced in the corresponding plane loaded frame by the load, $F_{y}=-1$, at B. This latter frame is called the conjugate frame and is the three hinged portal shown in Fig. 6a. The bending moments due to the unit load are easily computed and are shown in Fig. 6b. The diagram showing the bending moments of the conjugate frame is at the same time the influence line for the bending moment produced at the section $\mathrm{B}$ of the space loaded frame by transverse loads $F_{\mathrm{z}}$.

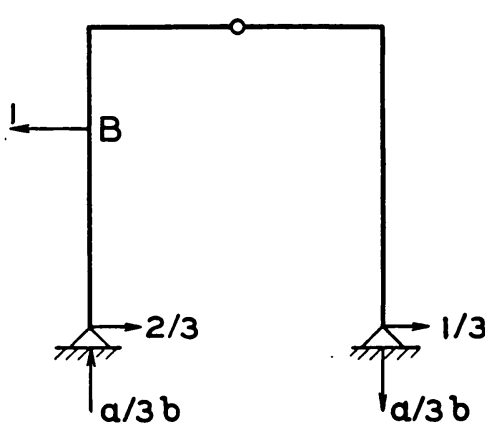

FIG. 6a.

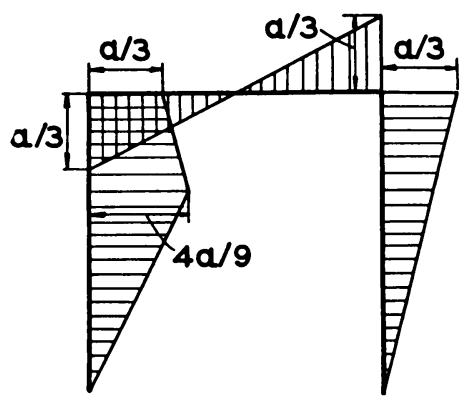

FIG. 6b.

Similarly, the influence line for the torque $M_{x}$ produced at the section B of the space loaded frame by transverse loads $F_{z}$ can be obtained as the bending moment diagram of the conjugate frame due to the load $F_{x}=-1$ at $\mathrm{B}$. Finally, the influence line for the shear $R_{z}$ produced at the section $\mathrm{B}$ of the space loaded frame by transverse loads $F_{z}$ is found as the bending moment diagram of the conjugate frame due to the couple $C_{z}=-1$ at $\mathrm{B}$.

4. Analogy between kinematics of plane loaded and statics of space loaded frames. For a plane loaded frame the displacements $u_{z}, \theta_{x}, \theta_{y}$ vanish and the distortions $G_{z}, g_{z}, H_{x}, h_{x}, H_{y}, h_{y}$ are zero. For a space loaded frame the loads $F_{x}, f_{x}, F_{y}, f_{y}, C_{z}, c_{z}$ and the stress resultants $R_{x}, R_{y}, M_{z}$ vanish. The remaining equations (2) for the members of the plane loaded frame then are found to correspond exactly to the remaining equations (1) for the members of the space loaded frame according to Table III.

The relations between the displacements of the plane loaded frame at both sides of the angle joint of Fig. 3 are easily seen to correspond to the relations between the stress resultants of the space loaded frame. Furthermore, the 
correspondence between the various types of supports and links shown in Table II is valid also in the present case where we are concerned with the kinematics of the plane loaded frame and the statics of the space loaded frame.

TABLE III

\begin{tabular}{|c|c|}
\hline Kinematics of Plane Loaded Frame & Statics of Space Loaded Frame \\
\hline 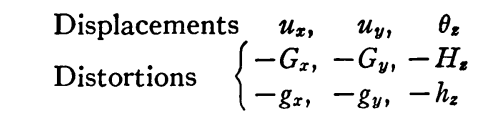 & $\begin{array}{l}\text { Stress resultants } M_{x}, M_{y}, R_{z} \\
\text { Loads }\left\{\begin{array}{lll}C_{x}, & C_{y}, & F_{z} \\
c_{x}, & c_{y}, & f_{z}\end{array}\right.\end{array}$ \\
\hline
\end{tabular}

The analogy established in this section can

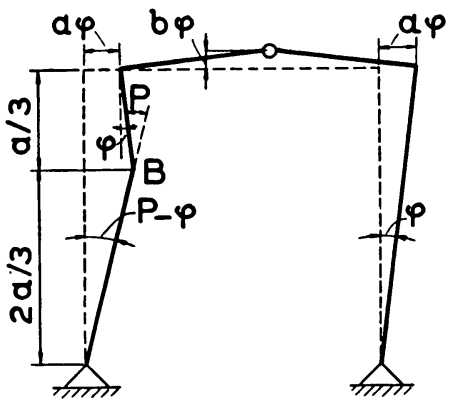

FIG. 7. be used to find the stress resultants of a statically determinate space loaded frame by determining the displacements of the conjugate plane loaded frame. Let us consider for instance the frame in Fig. 5 carrying a transverse load $F_{z}=P$ at $\mathrm{B}$. The stress resultants produced by this load can be found as the displacements of the conjugate frame produced by the bend $H_{z}=-P$ at $\mathrm{B}$. The general trend of these displacements is shown in Fig. 7. Relative to the right hand support, we obtain for the transverse displacement at $\mathrm{B}: u_{y}=4 a \varphi / 3$.

Relative to the left hand support, we have for the transverse displacement at the same point: $u_{y}=2 a(P-\varphi) / 3$. By equating these expressions and solving for $\varphi$, we find that $\varphi=P / 3$. The displacements $u_{x}, u_{y}, \theta_{z}$ of the conjugate frame are thus known. They correspond to the stress resultants $M_{x}, M_{y}, R_{z}$ of the space loaded frame, the distribution of which is shown in Figs. 8 a-c.

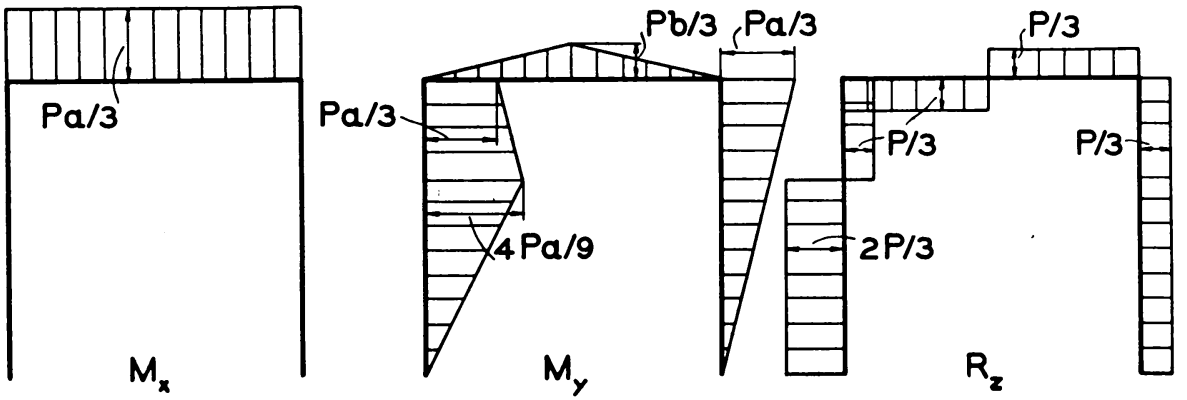

FIG. 8a-c.

5. Elastic deformations of space loaded frames. Indeterminate space loaded frames. When the stress resultants of a space loaded frame have been 
determined by the method given in the preceding section, the analogy of $\S 3$ can be used in order to find the elastic deformations of the space loaded frame. For example, let us consider again the frame in Fig. 5, carrying a transverse load $F_{z}=P$ at $\mathrm{B}$. The stress resultants have been determined in the preceding section and are shown in Fig. 8. Equations (3) furnish the distortions produced by these stress resultants: $h_{x}=M_{x} / \beta_{x}, h_{y}=M_{y} / \beta_{y}, g_{z}=R_{z} / \alpha_{z}$. According to the analogy of $\S 3$, the displacements $\theta_{x}, \theta_{y}, u_{z}$ corresponding to these distortions can be found as the stress resultants $R_{x}, R_{y}, M_{z}$ of the conjugate frame carrying the loads $f_{x}=-h_{x}, f_{y}=-h_{y}, c_{z}=-g_{z}$. In most cases the influences of the shear $R_{z}$ on the deformations can be neglected, and consequently in the conjugate frame the distributed couples $c_{z}$ need not be considered. The loads on the conjugate frame then consist of the axial loads shown in Fig. 9a and the transverse loads shown in Fig. 9b, it having been assumed that $\beta_{x}$ and $\beta_{y}$ have the same values for all members. The reactions can be computed easily and are indicated in Figs. 9a and 9b. If now, for instance, we wish to determine the deflection $u_{z}$ of the right hand corner $\mathrm{C}$ of the space loaded frame, we have only to compute the bending moment $M_{z}$ at the corresponding corner of the conjugate frame. We find that

$$
u_{z}=-\frac{P a^{3}}{162 \beta_{y}}\left[23-18 n^{3}+54 \gamma n\right],
$$

where $n=b / a$ and $\gamma=\beta_{y} / \beta_{x}$.

This method of computing elastic deformations enables us to carry out the stress analysis of indeterminate space loaded frames. Let us suppose, for instance, that the frame in Fig. 5 is given a further simple support at B and carries a transverse load $F_{z}=Q$ at $\mathrm{C}$. By establishing the condition for vanish-

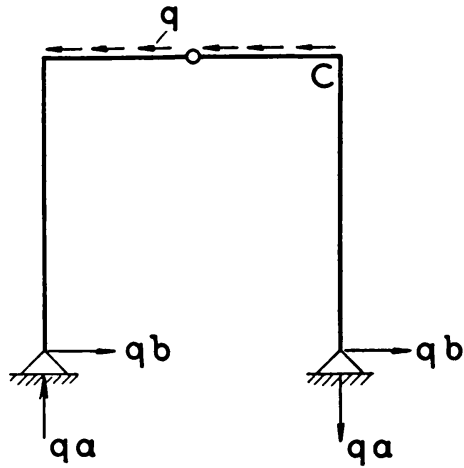

Fig. 9a. $q=P a / 3 \beta_{x}$.

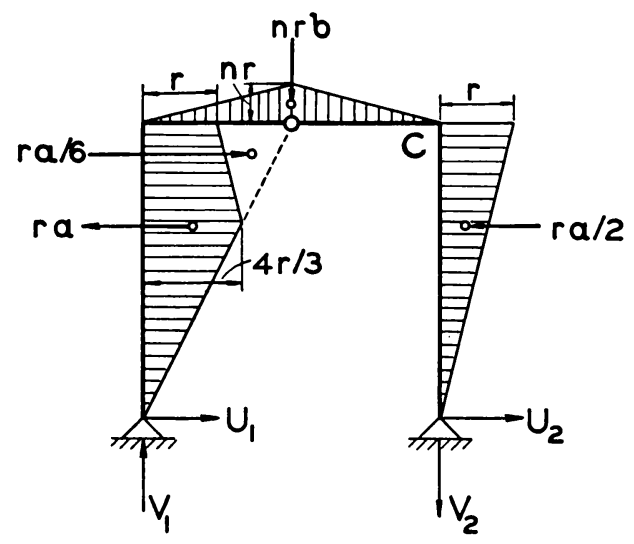

FIG. 9b. $n=b / a, r=P a / 3 \beta_{y}$,

$$
\begin{array}{ll}
U_{1}=\frac{r a}{54}\left(40+18 n^{3}\right), & U_{2}=\frac{r a}{54}\left(32-18 n^{3}\right) . \\
V_{1}=\frac{r a}{54 n}\left(23+27 n^{3}\right) & V_{2}=\frac{r a}{54 n}\left(23-27 n^{3}\right) .
\end{array}
$$


ing deflection at $B$ and applying Maxwell's law of reciprocal deflections in the usual way, we find the reaction at $\mathrm{B}$ in the form $R=-Q u_{C} / u_{B}$, where $u_{B}$ and $u_{C}$ denote the deflections which a unit transverse load $F_{z}=1$ at $\mathrm{B}$ produces at the points $\mathrm{B}$ and $\mathrm{C}$ respectively; $u_{C}$ can be obtained from (6) by setting $P=1 ; u_{B}$ can be computed as the bending moment $M_{z}$ at the point $\mathrm{B}$ of the conjugate frame, loaded according to Figs. 9a and 9b. With $P=1$ we find that

$$
u_{B}=\frac{a^{3}}{243 \beta_{y}}\left[32+18 n^{3}+54 \gamma n\right]
$$

and therefore

$$
R=\frac{3\left(23-18 n^{3}+54 \gamma n\right)}{2\left(32+18 n^{3}+54 \gamma n\right)}
$$

6. The inverse column analogy. The following method of determining the stress resultants of indeterminate space loaded frames is patterned after

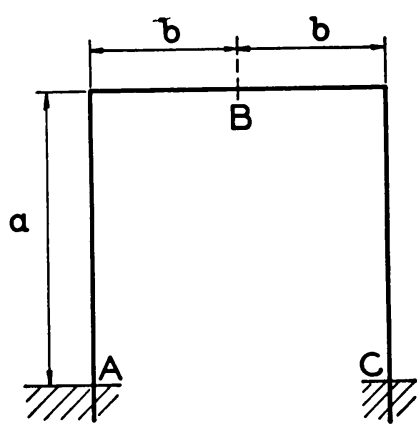

FIG. 10. the column analogy method of $\mathrm{H}$. Cross. ${ }^{3}$ In order to avoid lengthy computations which might obscure the essential feature of this new method, we shall consider the simple problem of the frame in Fig. 10 carrying a transverse load $F_{z}=P$ at B.

We suppose again that the stiffness factors $\beta_{x}$ and $\beta_{y}$ have the same values for all members. The frame then is symmetrical and is loaded symmetrically.

According to the analogy of $\S 4$, the torque $M_{x}$ and the bending moment $M_{y}$ of the frame can be obtained as the displacements $u_{x}$ and $u_{y}$ of the conjugate frame produced by the bend $H_{z}=-P$ at $\mathrm{B}$. Now the conjugate frame is entirely free. Since the system is symmetrical, it will possess a kinematically indeterminate displacement $v$ in the direction of the columns as shown in Fig. 11a. This indeterminate displacement in the plane loaded frame corresponds to the statically indeterminate torque in the columns of the space loaded frame.

From equations (2) we see that the distortions of the space loaded frame are given by $h_{x}=M_{x} / \beta_{x}, h_{y}=M_{y} / \beta_{y}$, or, by the analogy of $\S 4$, by $h_{x}=u_{x} / \beta_{x}$, $h_{y}=u_{y} / \beta_{y}$, where $u_{x}$ and $u_{y}$ are the displacements in the conjugate plane loaded frame. The analogy of $\$ 3$ then indicates that the longitudinal and transverse loads on the conjugate frame are given by $f_{x}=-u_{x} / \beta_{x}, f_{y}=-u_{y} / \beta_{y}$.

${ }^{3} \mathrm{H}$. Cross, The column analogy, Univ. Illinois Engineering Experiment Station, Bull. No. 215, 1930. 
The horizontal load is in equilibrium because of symmetry. The vertical load is shown in Fig. 11b, and is in equilibrium if

$$
v=\frac{P b}{4} \frac{n}{n+\gamma},
$$

where $n=b / a, \gamma=\beta_{y} / \beta_{x}$. Thus the torque $M_{x}$ in the left hand column is given by $M_{x}=u_{x}=v$. Also, the bending moment $M_{y}$ at $\mathrm{B}$ is given by

$$
M_{\nu}=-(v-P b / 2)=\frac{P b}{4} \frac{2 \gamma+n}{\gamma+n} .
$$

The procedure outlined above is equivalent to the following procedure. We suppose that the conjugate frame is embedded in an elastic jelly which

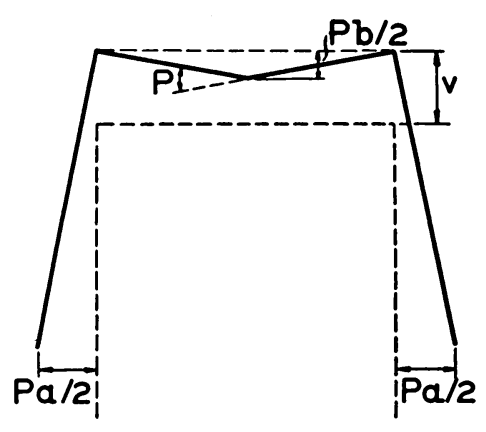

FIG. 11a.

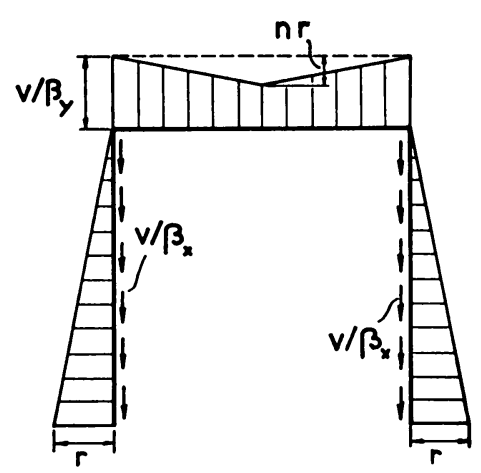

FIG. $11 b . n=b / a, r=P a / 2 \beta_{y}$.

offers resistance to the displacement of the frame in such a way that an element $d s$ with displacements $u_{x}$ and $u_{y}$ will meet with a resistance consisting of longitudinal and transverse forces of magnitudes $u_{x} d s / \beta_{x}$ and $u_{y} d y / \beta_{y}$, respectively. We then determine the displacements of this elastically supported frame, and the longitudinal displacement $u_{x}$ then gives the torque $M_{x}$ in the space loaded frame, while the transverse displacement $u_{y}$ gives the bending moment $M_{y}$. The above method of procedure is clearly seen to be the counterpart of the column analogy of $\mathrm{H}$. Cross; it will be called the inverse column analogy.

The inverse column analogy furnishes a simple method of determining the influence lines of an indeterminate space loaded frame. For example, let us consider the influence line for the bending moment $M_{y}$ which transverse loads $F_{z}$ produce at the section B of the symmetric frame in Fig. 10. Following the line of approach of $\$ 3$, we consider the conjugate frame loaded by a transverse force $F_{y}=-1$ at $\mathrm{B}$ and supported elastically in accordance with the inverse column analogy. Since this frame is symmetrical and loaded symmetrically, the displacements are of the type indicated in Fig. 12a. The forces 
which the elastic support exerts on the frame are shown in Fig. 12b. From the condition of equilibrium for the vertical forces, we find that

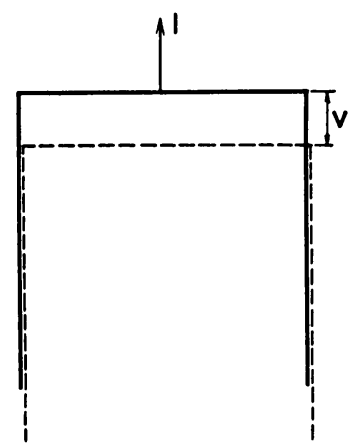

Fig. 12a.

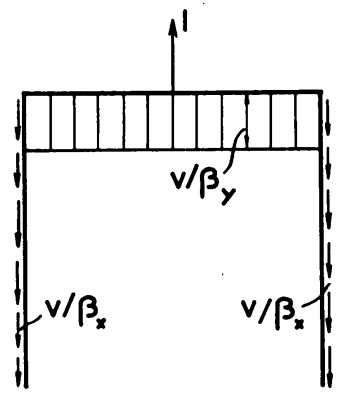

Fig. 12b.

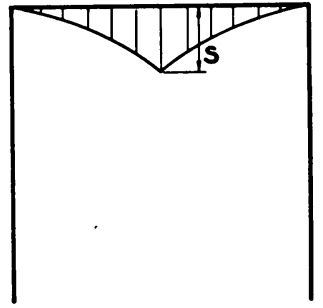

FIG. 12c. $s=v a b / \beta_{x}+v b^{2} / \beta_{y}$.

$$
v=\frac{\beta_{y}}{2 b} \frac{n}{n+\gamma},
$$

where again $n=b / a$ and $\gamma=\beta_{y} / \beta_{x}$. The bending moment diagram of this elastically supported frame (Fig. 12c) is at the same time the desired influence line of the space loaded frame. 